\title{
On the singularities of gravity in the presence of non-minimally coupled scalar fields
}

\author{
L.R. Abramo ${ }^{1}$, L. Brenig ${ }^{2}$, E. Gunzig ${ }^{2,3}$, Alberto $\mathrm{Saa}^{2,4}$ 因 \\ 1) Instituto de Física, Universidade de São Paulo, CP 66318, 05315-970 São Paulo, SP, Brazil \\ 2) RggR, Université Libre de Bruxelles, CP 231, 1050 Bruxelles, Belgium. \\ 3) Instituts Internationaux de Chimie et de Physique Solvay, CP 231, 1050 Bruxelles, Belgium. \\ 4) IMECC-UNICAMP, C.P. 6065, 13081-970 Campinas, SP, Brazil.
}

\begin{abstract}
We investigate the robustness of some recent results obtained for homogeneous and isotropic cosmological models with conformally coupled scalar fields. For this purpose, we investigate anisotropic homogeneous solutions of the models described by the action

$$
S=\int d^{4} x \sqrt{-g}\left\{F(\phi) R-\partial_{a} \phi \partial^{a} \phi-2 V(\phi)\right\}
$$

with general $F(\phi)$ and $V(\phi)$. We show that such a class of models leads generically to geometrical singularities if for some value of $\phi, F(\phi)=0$, rendering previous cosmological results obtained for the conformal coupling case highly unstable. We show that stable models can be obtained for suitable choices of $F(\phi)$ and $V(\phi)$. Implications for other recent results are also discussed.

98.80.Cq, $98.80, \mathrm{Bp}, 98.80 . \mathrm{Hw}$
\end{abstract}

Typeset using REVTEX

*e-mails: abramo@fma.if.usp.br, lbrenig@ulb.ac.be, egunzig@ulb.ac.be, asaa@ime.unicamp.br 


\section{INTRODUCTION}

Recently [1,2], we have investigated the dynamics of homogeneous and isotropic solutions of the cosmological model described by the action:

$$
S=\int d^{4} x \sqrt{-g}\left\{F(\phi) R-\partial_{a} \phi \partial^{a} \phi-2 V(\phi)\right\}
$$

with $F(\phi)=1-\frac{1}{6} \phi^{2}$, corresponding to the so-called conformal coupling, and $V(\phi)=$ $\frac{m}{2} \phi^{2}-\frac{\Omega}{4} \phi^{4}$. Some novel dynamical behaviors were identified: superinflation regimes, a possible avoidance of big-bang singularities through classical birth of the universe from empty Minkowski space, spontaneous entry into and exit from inflation, and a cosmological history suitable for describing quintessence. Since one of the proposals of inflationary models is to describe our universe without finely-tuned parameters, these results would be viable only if they are robust against small perturbations in initial conditions and in the model itself. This is the question to be addressed here.

We study the robustness of our previous results by taking two generalizations of the model considered previously: we relax the isotropy requirements (perturbations in the initial conditions) and we consider a general coupling $F(\phi)$ (perturbations in the model parameters). Models with more general $F(\phi)$ have been considered recently [3]. Our results show that the model is not robust. Its main properties are radically changed, even for small disturbances in initial conditions and in the model itself, due to the appearance of real, gravitational singularities that are dynamically unavoidable in general. The singularities are, essentially, of two types. The first one corresponds to the hypersurfaces $F(\phi)=0$. It is not present in the isotropic case, and it implies that all previous homogeneous and isotropic solutions passing from the $F(\phi)>0$ to the $F(\phi)<0$ region are extremely unstable against anisotropic perturbations. The second type of singularity corresponds to $F_{1}(\phi)=0$, with

$$
F_{1}(\phi)=F(\phi)+\frac{3}{2}\left(F^{\prime}(\phi)\right)^{2},
$$

and it is present even for the homogeneous and isotropic cases. Although for small deviations of the conformal coupling the latter singularities are typically very far from the region of 
interest, in the general case they can alter qualitatively the global dynamics of the model due to restrictions that it imposes on the phase space. Again, the persistence of some of our previously described results, in particular the ones concerning heteroclinic and homoclinic solutions, are challenged.

Both kinds of singularities have already been described before. To the best of our knowledge, Starobinski [4] was the first to identify the singularity corresponding to the hypersurfaces $F(\phi)=0$, for the case of conformally coupled anisotropic solutions. Futamase and co-workers [5] identified both singularities in the context of chaotic inflation in $F(\phi)=1-\xi \phi^{2}$ theories (See also [6]). The first singularity is always present for $\xi>0$ and the second one for $0<\xi<1 / 6$. Our conclusions are, however, more general since we treat the case of general $F(\phi)$ and our results are based on the analysis of true geometrical invariants. Our main result is that the system governed by (11) is generically singular on both hypersurfaces $F(\phi)=0$ and $F_{1}(\phi)=0$. Here, generically means that it is possible to construct non-singular models if one fine-tunes $F(\phi)$ and $V(\phi)$, as we will show below.

One can advance that there are some geometrically special regions on the phase space of the model in question by a very simple analysis of the equations derived from the action (四). They are the Klein-Gordon equation

$$
\square \phi-V^{\prime}(\phi)+\frac{1}{2} F^{\prime}(\phi) R=0,
$$

and the Einstein equations

$$
\begin{aligned}
F(\phi) G_{a b} & =\left(1+F^{\prime \prime}(\phi)\right) \partial_{a} \phi \partial_{b} \phi \\
& -\frac{1}{2} g_{a b}\left[\left(1+2 F^{\prime \prime}(\phi)\right) \partial_{c} \phi \partial^{c} \phi+2 V(\phi)\right]-F^{\prime}(\phi)\left(g_{a b} \square \phi-\nabla_{a} \phi \nabla_{b} \phi\right) .
\end{aligned}
$$

We will consider here the simplest anisotropic homogeneous cosmological model, the Bianchi type I, whose spatially flat metric is given by

$$
d s^{2}=-d t^{2}+a^{2}(t) d x^{2}+b^{2}(t) d y^{2}+c^{2}(t) d z^{2} .
$$

The dynamically relevant quantities here are 


$$
H_{1}=\frac{\dot{a}}{a}, \quad H_{2}=\frac{\dot{b}}{b}, \quad \text { and } H_{3}=\frac{\dot{c}}{c}
$$

For such a metric and a homogeneous scalar field $\phi=\phi(t)$, after using the Klein-Gordon Eq. (3), Eq. (1) can be written as

$$
\begin{aligned}
F(\phi) G_{00} & =\frac{1}{2} \dot{\phi}^{2}+V(\phi)-F^{\prime}(\phi)\left(H_{1}+H_{2}+H_{3}\right) \dot{\phi}, \\
\frac{1}{a^{2}} F(\phi) G_{11} & =\frac{1+2 F^{\prime \prime}(\phi)}{2} \dot{\phi}^{2}-V(\phi)-F^{\prime}(\phi)\left(H_{1} \dot{\phi}+V^{\prime}(\phi)-\frac{F^{\prime}(\phi)}{2} R\right), \\
\frac{1}{b^{2}} F(\phi) G_{22} & =\frac{1+2 F^{\prime \prime}(\phi)}{2} \dot{\phi}^{2}-V(\phi)-F^{\prime}(\phi)\left(H_{2} \dot{\phi}+V^{\prime}(\phi)-\frac{F^{\prime}(\phi)}{2} R\right), \\
\frac{1}{c^{2}} F(\phi) G_{33} & =\frac{1+2 F^{\prime \prime}(\phi)}{2} \dot{\phi}^{2}-V(\phi)-F^{\prime}(\phi)\left(H_{3} \dot{\phi}+V^{\prime}(\phi)-\frac{F^{\prime}(\phi)}{2} R\right) .
\end{aligned}
$$

It is quite simple to show that Eqs. (8)-(10) are not compatible, in general, on the hypersurface $F(\phi)=0$. Subtracting (9) and (10) from (8) we have, on such hypersurface, respectively,

$$
F^{\prime}(\phi)\left(H_{1}-H_{2}\right) \dot{\phi}=0 \text {, and } \quad F^{\prime}(\phi)\left(H_{1}-H_{3}\right) \dot{\phi}=0
$$

Hence, they cannot be fulfilled in general for anisotropic metrics. As it will be shown, it indeed corresponds to an unmovable (in the Painlevé sense [7]) geometrical singularity which cannot be prevented in general by requiring that $F^{\prime}(\phi)=0$ or $\dot{\phi}=0$ on the hypersurface.

As to the second singularity we have, after taking the trace of the Einstein equations, that:

$$
R=R(\phi, \dot{\phi})=\frac{1}{F_{1}(\phi)}\left(4 V(\phi)+3 V^{\prime}(\phi) F^{\prime}(\phi)-\left(1+F^{\prime \prime}(\phi)\right) \dot{\phi}^{2}\right)
$$

Inserting Eq. (12) in the Klein-Gordon Eq. (3), one can see that it contains terms which are singular for $F_{1}(\phi)=0$. Again, as we will see, this corresponds to an unmovable geometrical singularity, and it cannot be eliminated, in general, by demanding that $F^{\prime}(\phi)=0$ on the hypersurface $F_{1}(\phi)=0$. In both the hypersurfaces $F(\phi)=0$ and $F_{1}(\phi)=0$ the Cauchy problem is ill-posed, since one cannot choose general initial conditions.

The hypersurfaces $F(\phi)=0$ and $F_{1}(\phi)=0$ also prevent the global definition of an Einstein frame for the action (1), defined by the transformations 


$$
\begin{aligned}
\tilde{g}_{a b} & =F(\phi) g_{a b} \\
\left(\frac{d \tilde{\phi}}{d \phi}\right)^{2} & =\frac{F_{1}(\phi)}{2 F(\phi)^{2}}
\end{aligned}
$$

It is well known that in the Einstein frame the Cauchy problem is well posed. Again, the impossibility of defining a global Einstein frame shed some doubts about the general Cauchy problem. Moreover, the standard perturbation theory for helicity-2 and helicity-0 excitations, derived directly from Eqs. (13)-(14), fails in both hypersurfaces [3].

\section{THE SINGULARITIES}

In order to check the geometrical nature of these singular hypersurfaces, let us consider the Einstein Eqs. (7)-(10) in detail. For the metric (5), we have the following identities

$$
\begin{aligned}
G_{00} & =H_{1} H_{2}+H_{2} H_{3}+H_{1} H_{3}, \\
G_{11} & =a^{2}\left(\dot{H}_{1}+H_{1}\left(H_{1}+H_{2}+H_{3}\right)-\frac{1}{2} R\right), \\
G_{22} & =b^{2}\left(\dot{H}_{2}+H_{2}\left(H_{1}+H_{2}+H_{3}\right)-\frac{1}{2} R\right), \\
G_{33} & =c^{2}\left(\dot{H}_{3}+H_{3}\left(H_{1}+H_{2}+H_{3}\right)-\frac{1}{2} R\right), \\
R & =2\left(\dot{H}_{1}+\dot{H}_{2}+\dot{H}_{3}+H_{1}^{2}+H_{2}^{2}+H_{3}^{2}+H_{1} H_{2}+H_{2} H_{3}+H_{1} H_{3}\right) .
\end{aligned}
$$

After using expressions (15) and introducing the new dynamical variables $p=H_{1}+H_{2}+H_{3}$, $q=H_{1}-H_{2}$, and $r=H_{1}-H_{3}$, Einstein Eqs. can be cast in the form

$$
\begin{aligned}
E(\phi, \dot{\phi}, p, q, r)= & -\frac{1}{3} F(\phi)\left(p^{2}+q r-q^{2}-r^{2}\right)+\frac{\dot{\phi}^{2}}{2}+V(\phi)-p F^{\prime}(\phi) \dot{\phi}=0, \\
\dot{q}= & -\left(p+\frac{F^{\prime}(\phi)}{F(\phi)} \dot{\phi}\right) q, \\
\dot{r}= & -\left(p+\frac{F^{\prime}(\phi)}{F(\phi)} \dot{\phi}\right) r \\
-2 F_{1}(\phi) \dot{p}= & \left(F(\phi)+2 F^{\prime}(\phi)^{2}\right) p^{2}+\frac{3}{2}\left(1+2 F^{\prime \prime}(\phi)\right) \dot{\phi}^{2}-3 V(\phi)-3 F^{\prime}(\phi) V^{\prime}(\phi) \\
& -p \dot{\phi} F^{\prime}(\phi)+\left(F(\phi)+F^{\prime}(\phi)^{2}\right)\left(q^{2}+p^{2}-q r\right)
\end{aligned}
$$

Using the energy constraint (16), this last equation can be put in the form 


$$
\begin{aligned}
\dot{p}= & -\left(p+\frac{F^{\prime}(\phi)}{F(\phi)} \dot{\phi}\right) p+3 \frac{F(\phi)+\frac{1}{2}\left(F^{\prime}(\phi)\right)^{2}}{F(\phi) F_{1}(\phi)} V(\phi) \\
& -\frac{3}{4}\left(\frac{2 F(\phi) F^{\prime \prime}(\phi)-\left(F^{\prime}(\phi)\right)^{2}}{F(\phi) F_{1}(\phi)}\right) \dot{\phi}^{2}+\frac{3}{2} \frac{F^{\prime}(\phi)}{F_{1}(\phi)} V^{\prime}(\phi) .
\end{aligned}
$$

The Klein-Gordon equation (3) reads simply

$$
\ddot{\phi}+p \dot{\phi}+V^{\prime}(\phi)-\frac{F^{\prime}(\phi)}{2} R(\phi, \dot{\phi})=0
$$

with $R(\phi, \dot{\phi})$ given by Eq. (12). The energy constraint, equation (16), is evidently compatible with the other ones. Indeed $E(\phi, \dot{\phi}, p, q, r)=0$ is an invariant surface since one has that

$$
\frac{d}{d t} E(\phi, \dot{\phi}, p, q, r)=-\left(2 p+\frac{F^{\prime}(\phi)}{F(\phi)} \dot{\phi}\right) E(\phi, \dot{\phi}, p, q, r)
$$

along solutions of Eqs. (17), (18), (20), and (21). Note that Eqs. (20) and (21) are decoupled from the equations for $\dot{q}$ and $\dot{r}$. Equations (17) and (18) are, hence, linear first order equations, and they could be easily integrated after the solutions of (20) and (21) have been found. Moreover, since one has $r \dot{q}-q \dot{r}=0, q(t) / r(t)$ is a constant of motion fixed only by the initial conditions. Suppose the initial ratio is $q(0) / r(0)=\gamma$ : this would imply that $\left(H_{1}-H_{2}\right)=\gamma\left(H_{1}-H_{3}\right)$ for all $t$, leading to, for instance, $c^{\gamma}(t) \propto a^{\gamma-1}(t) b(t)$ in the metric (5). This simplification is a consequence of the scalar character of our source field, and it does not suppose any loss of generality in our arguments.

A closer analysis of Eqs. (17)-(20) reveals the presence of the singularities. In general, the right-hand side of these equations diverge for $F(\phi)=0$ and for $F_{1}(\phi)=0$. One can check that these divergences are indeed related to real geometrical singularities by considering the Kretschman invariant $I=R_{a b c d} R^{a b c d}$, which for the metric (5) is given by

$$
I=4\left(\left(\dot{H}_{1}+H_{1}^{2}\right)^{2}+\left(\dot{H}_{2}+H_{2}^{2}\right)^{2}+\left(\dot{H}_{3}+H_{3}^{2}\right)^{2}+H_{1}^{2} H_{2}^{2}+H_{1}^{2} H_{3}^{2}+H_{2}^{2} H_{3}^{2}\right) .
$$

As one can see, $I$ is the sum of non negative terms. Moreover, any divergence of the variables $H_{1}, H_{2}, H_{3}$, or of their time derivatives, would suppose a divergence in $I$, characterizing a real geometrical singularity. Since the relation between the variables $p, q, r$, and $H_{1}, H_{2}, H_{3}$ is linear, any divergence of the first, or of their time derivative, will suppose a divergence in $I$. 
Suppose, now, that $F\left(\phi_{0}\right)=0$, and that $F(\phi)$ is (real) analytical for $\phi=\phi_{0}$. In this case, $F^{\prime}(\phi) / F(\phi)$ diverges as $\left(\phi-\phi_{0}\right)^{-1}$ near $\phi_{0}$ for nonvanishing $q$ and $r$, rendering $\dot{q}$ and $\dot{r}$ divergent by (17) and (18), unless $p$ also diverges in order to keep $\left(p+\frac{F^{\prime}(\phi)}{F(\phi)} \dot{\phi}\right)$ finite on $\phi_{0}$. In both cases, $I$ diverges. There is no dynamical restriction to ensure that $\dot{\phi}$ vanishes on $\phi_{0}$ - it can take any value compatible with the energy constraint (16). Indeed, the latter implies that on $\phi_{0}$

$$
\frac{\dot{\phi}^{2}}{2}-p F^{\prime}\left(\phi_{0}\right) \dot{\phi}+V\left(\phi_{0}\right)=0
$$

There is no way of having $\dot{\phi}=0$ on $\phi_{0}$, unless $V\left(\phi_{0}\right)=0$, and even in this case, $\dot{\phi}=p F^{\prime}\left(\phi_{0}\right)$ is also possible. Note that the hypothesis of $F(\phi)$ analytical at $\phi_{0}$ is not a necessary one. For any differentiable function $F(\phi)$ with a zero in $\phi_{0}$ one has $|F(\phi)|=\left|\int_{\phi_{0}}^{\phi} F^{\prime}(s) d s\right| \leq k\left|\phi-\phi_{0}\right|$, with $k=\max _{s \in\left[\phi_{0}, \phi\right]}\left|F^{\prime}(s)\right|$, implying that $\left|F^{\prime}(\phi) / F(\phi)\right| \geq\left|\phi-\phi_{0}\right|^{-1}\left|F^{\prime}(\phi)\right| / k$. Since $F^{\prime}(\phi)$ is assumed to be continuous, the last ratio tends to 1 when $\phi \rightarrow \phi_{0}$, implying the divergence of $F^{\prime}(\phi) / F(\phi)$ in that limit.

Now, let us suppose $F_{1}\left(\phi_{1}\right)=0$. If $F^{\prime}\left(\phi_{1}\right) \neq 0$, the right-hand side of Eq. (20) diverges. The vanishing of $F^{\prime}\left(\phi_{1}\right)$ implies, by (2), that $F\left(\phi_{1}\right)=0$, and the arguments of the last paragraph can be repeated.

A singularity-free model can be constructed by demanding that $F\left(\phi_{0}\right)=F^{\prime}\left(\phi_{0}\right)=0$, by choosing a $V(\phi)$ that goes to 0 at a proper rate when $\phi \rightarrow \phi_{0}$, and by demanding that $F_{1}(\phi)$ have no other zeros than the ones of $F(\phi)$. Models for which $F(\phi)=\zeta \phi^{2 n}$ and $V(\phi)=\alpha \phi^{2(2 n-1)}+$ high order terms, for instance, fulfill these requirements. However, such a highly fine-tuned class of model is of no physical interest here, since it does not contain $F(\phi)>0$ and $F(\phi)<0$ regions and consequently has no solution for which the effective gravitational constant $G_{\text {eff }}$ changes it sign along the cosmological history. The stability of such solutions were the starting point of the analyses of the pioneering work [4] and of the present one as well. Note that by Eq. (2), models with an $F(\phi)<0$ region will allways have singularities of the type $F_{1}(\phi)=0$. This fact shall be taken into account to better understand the recently proposed dynamical stability of the $F(\phi)<0$ region $[\mathbb{8}]$. 


\section{CONCLUSION}

The singularities described in the precedent section imply that the model presented in [1,2] is not robust, since our main conclusions were a consequence of very especial initial conditions. For instance, all homogeneous and isotropic solutions crossing the $F(\phi)=0$ hypersurface are extremely unstable against anisotropic perturbations. By Eqs. (17) and (18), any deviation from perfect isotropy (expressed by nonvanishing $q$ and $r$ variables) for these solutions, however small, will lead catastrophically to a geometrical singularity. Many of the novel dynamical behaviors presented in [1,2] depend on these solutions. This is the case, for instance, of some solutions exhibiting superinflation regimes. The heteroclinic and homoclinic solutions identified in [1,2] can cross the $F(\phi)=0$ hypersurface and, hence, they also suffer the same instability against anisotropic perturbations. The homoclinic solutions were considered as candidates to describe a non-singular cosmological history, with the big-bang singularity being avoided through a classical birth of the universe from empty Minkowski space. Apart from $F(\phi)=0$ singularities, these solutions are also affected by the singularities of type $F_{1}(\phi)=0$. Suppose that the conformal coupling is disturbed by a very small negative term: $F(\phi)=1-\left(\frac{1}{6}-\epsilon\right) \phi^{2}$. The $F_{1}(\phi)=0$ singularities will be near the $\phi= \pm 1 / \sqrt{\epsilon}$ hypersurfaces. Although they are located far from the $F(\phi)=0$ regions, they alter the global structure of the phase-space. In this case, they restrict the existence of homoclinics, rendering a non-singular cosmological history more improbable.

The singularities do not affect the conclusions obtained by considering solutions inside the $F(\phi)>0$ region. The asymptotic solutions presented in [2], for instance, are still valid. The conclusion that for large $t$ the dynamics of any solution (inside $F(\phi)>0$ ) tends to an infinite diluted matter dominated universe remains valid. Moreover, for small anisotropic deviations ( $q$ and $r$ small in comparision with $p$ ), Eqs. (17) and (18) allow us to conclude that solutions inside $F(\phi)>0$, for large $t$, approach exponentially isotropic matter-dominated universe. 


\section{ACKNOWLEDGMENTS}

The authors wish to thank G. Esposito-Farese for previous discussions concerning the Cauchy problem for models with $F(\phi)=0$, and Profs. Albert and Anny Sanfeld for the warm hospitality in Mallemort, France, where this work was initiated. They also acknowledge the financial support from the EEC (project HPHA-CT-2000-00015), from OLAM Fondation pour la Recherche Fondamentale (Belgium), from Fondation Science et Environnement (France), and from CNPq (Brazil). 


\section{REFERENCES}

[1] E. Gunzig, A. Saa, L. Brenig, V. Faraoni, T.M. Rocha Filho, and A. Figueiredo, Phys. Rev. D63, 067301 (2001).

[2] A. Saa, E. Gunzig, L. Brenig, V. Faraoni, T.M. Rocha Filho, and A. Figueiredo, Int. J. Theor. Phys. 40, 2295 (2001).

[3] G. Esposito-Farese and D. Polarski, Phys. Rev. D63, 063504 (2001).

[4] A. A. Starobinski, P. Astron. Zh. 7, 67 (1981) [Sov. Astron. Lett. 7, 36 (1981)].

[5] T. Futamase and K. Maeda, Phys. Rev. D39, 399 (1989); T. Futamase, T. Rothman, and R. Matzner, Phys. Rev. D39, 405 (1981).

[6] S. Deser, Phys. Lett. 134B, 419 (1984); Y. Hosotani, Phys. Rev. D32, 1949 (1985); O. Bertolami, Phys. Lett. 186B, 161 (1987).

[7] See, for instance, Painlevé transcendents: their asymptotics and physical applications, Nato Advanced Research Workshop held in Quebec, September 1990. Edited by D. Levi and P. Winternitz, NATO Advanced Science Institutes Series B, Physics, vol. 278, Plenum Press (NY), 1992.

[8] L.R. Abramo, L. Brenig, and E. Gunzig, On the stability of gravity in the presence of a non-minimally coupled scalar field, gr-qc/0205022, to be published. 